\title{
Mechanical Properties of 3D Isotropic Anti-Tetrachiral Metastructure
}

\author{
Re Xia, Xiaoke Song, Lijuan Sun, Wenwang Wu,* Chuanlei Li, Tianbao Cheng, \\ and Guian Qian*
}

Chiral metastructures consisting of ring (polygonal or cubic) nodes and elastic bending ligaments exhibit excellent design flexibility for compliant structures. In this paper, based on rigid cubic node rotation and the kinematic geometrical relations of a three-dimensional (3D) isotropic anti-tetrachiral structure, an analytical expression for the modulus of the 3D isotropic anti-tetrachiral structure is derived from strain energy analysis. The nylon powder Selected Laser Sintering (SLS) additive manufacturing technique is employed for fabricating two types of 3D isotropic anti-tetrachiral specimens with different geometrical parameters; then comparison between experiments, finite element analysis (FEA), and theoretical studies is performed for verifying the analytical formula. The mechanical properties of 3D isotropic anti-tetrachiral structures can be tuned with two independent dimensionless geometrical parameters. The proposed 3D anti-tetrachiral structures can be employed for designing advanced structures against impact damages, realizing flexibility of industrial components, and optimizing the vibration attenuation abilities of engineering structures.

enhanced fracture toughness, enhanced porosity variation when stretched or compressed, enhanced energy absorption. Thus, auxetic materials can be applied for designing innovative multifunctional structures, such as: body armor, packing material, knee and elbow pads, robust shock absorbing material, and sponge mops. As the simple artificial auxetic structures, 2D cellular auxetic materials are featured by flexibility of interconnecting ribs or the rotation of nodes. Based on the unit cell deformation mechanism, auxetic cellular structures can be classified into: 1) re-entrant structures, where the diagonal ribs move in such a way that leads to auxetic effect in the other direction different from loading direction; 2) chiral structure, where the coupled deformation of node rotation and ligament bending gives rise to auxetic behavior; and 3) rigid (semi-rigid) rotating structures,

\section{Introduction}

Auxetic material (or metastructures) with negative Poisson ratio (NPR) is a special type of material which can expand its width along the directions perpendicular to its loading direction. The main deformation features and mechanical benefits of auxetic materials can be summarized as: enhanced indentation resistance,

Dr. R. Xia, Dr. X. Song

Key Laboratory of Hydraulic Machinery Transients (Wuhan University)

Ministry of Education

Wuhan 430072, P.R. China

Dr. L. Sun

State Key Laboratory for Nonlinear Mechanics (LNM)

Institute of Mechanics

Chinese Academy of Sciences

Beijing 100080, P.R. China

Dr. W. Wu, Dr. C. Li, Dr. T. Cheng

Institute of Advanced Structure Technology

Beijing Institute of Technology

Beijing 100081, P.R. China

E-mail: wuwenwang@bit.edu.cn

Dr. G. Qian

Laboratory for Nuclear Materials

Nuclear Energy and Safety Department

Paul Scherrer Institute

Villigen PSI 5232, Switzerland

E-mail: guian.qian@psi.ch

DOI: 10.1002/pssb.201700343 where auxetic behavior is obtained from the rotation of rigid polygons joined with each other through hinges, such as rotating squares, rotating rectangles, rotating parallelogram and rhombi, rotating triangles, and rotating tetrahedral. ${ }^{[1]}$ The node rotation of chiral structures will introduce additional parameters for tuning the mechanical properties of the chiral unit cell. ${ }^{[2]}$ Depending on the spatial relations between ligaments and nodes, structures with nodes on the opposite sides of the ligament are called chiral systems, while structures with nodes on the same side of the ligament are called anti-chiral systems. ${ }^{[3]}$ According to the number of ligaments connecting to the same node, different types of chiral systems are proposed, such as: hexachiral, tetrachiral, antitetrachiral, trichiral, and anti-trichiral structures. ${ }^{[3]}$

Based on kinematic deformation relations between the circular nodes and ligaments, Prall and Lakes ${ }^{[4]}$ investigated the in-plane mechanical properties of 2D isotropic hexachiral lattice structure for the first time. Afterwards, Alderson et al. ${ }^{[2]}$ studied the in-plane elastic constants of 3-, 4- and 6-connected chiral and anti-chiral honeycombs with similar deformation assumption. Making use of Castigliano's second theorem, Mousanezhad et al. ${ }^{[5]}$ derived analytical expression for the inplane elastic modulus of trichiral, tetrachiral and anti-tetrachiral structures, and compared the results from analytical formulas and finite element analysis. Following Prall and Lakes assumption, Chen et al. ${ }^{[6]}$ studied the in-plane mechanical properties of anisotropic anti-tetrachiral lattices with different ligaments along $x$ and $y$ directions, and it is demonstrated that 
the anisotropy ratio are strongly related to the unit cell lattice geometry parameters. Spadoni et al. ${ }^{[7,8]}$ proposed a micropolar continuum model for the elastic constants of hexachiral structures, and deformable ring node model are proposed and compared with rigid node model results. Through comparison between beam-lattice model and continuous model, Bacigalupoet al. ${ }^{[9]}$ derived the analytical formulas for the in-plane elastic constants of anti-tetrachiral cellular structures based on homogenization theory. Making use of non-local homogenization techniques, Bacigalupo and Gambarotta ${ }^{[10]}$ proposed a beamlattice micropolar equivalent continuum model for studying the mechanical properties of hexachiral and tetrachiral structures. Liu et al. ${ }^{[11]}$ developed a continuum theory for describing both the dilatation-rotation coupling and shear-rotation coupling deformation mechanism of chiral lattice structures, and derived the analytical formulas for the mechanical properties of chiral lattices as well. Based on micropolar theory, Chen et al. ${ }^{[12]}$ proposed analytical model for describing the constitutive relation for tetrachiral lattice structure, where 13 independent material constants are employed to represent the normal and higher order elastic tensors of the tetrachiral lattice structure. Li et al. ${ }^{[13]}$ proposed a tetrachiral and anti-tetrachiral hybrid stent with negative Poisson's ratio to reduce the shearing stress due to axial shortening effects. Wu et al. ${ }^{[14]}$ proposed the design of hierarchical anti-tetrachiral structures with square and circular nodes on different structural levels, and analytical models are proposed for describing its in-plane mechanical properties and auxetic deformation abilities. Besides auxetic properties, chiral composite structures exhibit excellent low frequency vibration attenuation, and sound absorption abilities. Liu et al. ${ }^{[15]}$ reviewed the in-plane elastic mechanical properties of chiral composite structures, and the bulk-rotational coupling mechanism of chiral structures is elaborated in details. Spadoni et al. ${ }^{[16,17]}$ studied the sound transmission reduction and vibration isolation properties of chiral truss-core sandwich beams. Abdeljaber et al. ${ }^{[18]}$ proposed an automated genetic algorithm based optimization approach for obtaining optimized chiral lattice configuration with broadband vibration control capabilities, and nine parameters governing the shape and grading of the lattice are considered in the optimization process, including the number of circular nodes, the thickness of the ligaments, and the characteristic angle, etc.

As to the engineering application of chiral structure, Scarpa et al. ${ }^{[19]}$ designed a hexachiral shape memory alloy deployable antenna, which shows great advantage in space saving. Similarly, Hassan et al. ${ }^{[20]}$ developed a shape memory alloy chiral deployable antenna manufacturing procedure. Spadoni and Ruzzene ${ }^{[8,21]}$ proposed an innovative chiral-core airfoils, and significant changes in the chord-wise bending compliance can be achieved through adjusting the chiral-core geometric parameters. Budarpuet al. ${ }^{[22]}$ proposed a chiral-core morphing airfoil with elliptical nodes, and the geometrical configuration of the chiral-core structure can be optimized to meet various morphing behaviors and global stiffness requirements, thus improving the aerodynamic performance of the airfoil. Ma et al. ${ }^{[23]}$ proposed the design of anti-tetrachiral vibration damper sandwich composite structures, where the cylinders are filled with the metal rubber particles (MRP) material. Static and dynamic vibration tests are performed to assess the performances of the integrated auxetic-MRP damper structures; it is found that the lightweight integrated auxetic damping panel exhibit higher damping and loading capability. Ha et al. ${ }^{[24-25]}$ proposed an innovative isotropic 3D tetrachiral metastructure, and studied its mechanical properties via finite element analysis, where the cubic nodes of the 3D tetrachiral metastructure are assumed as rigid nodules, and lattices connecting cubic nodes exhibit stretch-twist coupling deformation mechanism. It is found that both the effective Young's modulus and the effective shear modulus show convergence to a constant value with an increase of the cells in each side, and Poisson's ratio decreases from positive to negative values as the number of cells increases.

In this paper, based on rigid cubic node rotation and kinematic deformation relations of ligaments and nodes, analytical expression for the modulus of 3D isotropic antitetrachiral structure is derived from strain energy analysis, and comparison between experiments, FEA and theoretical studies

(a)

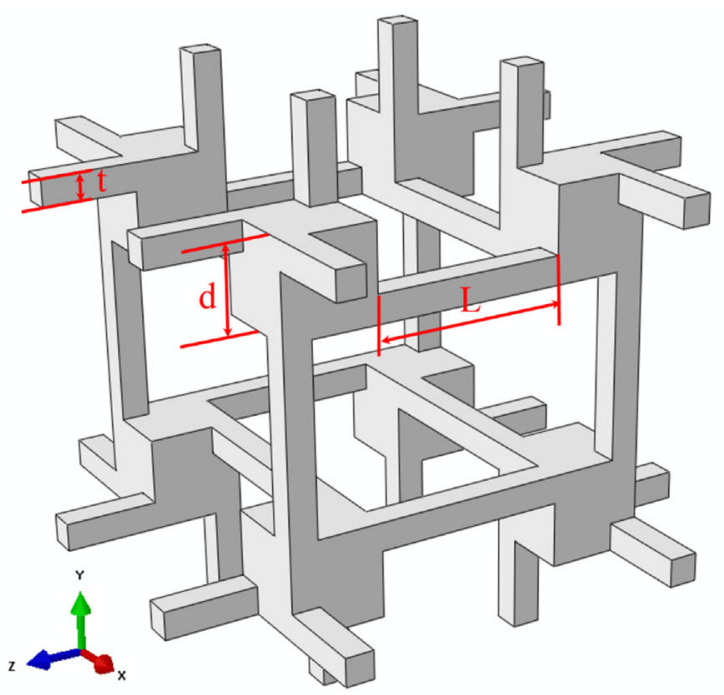

(b)

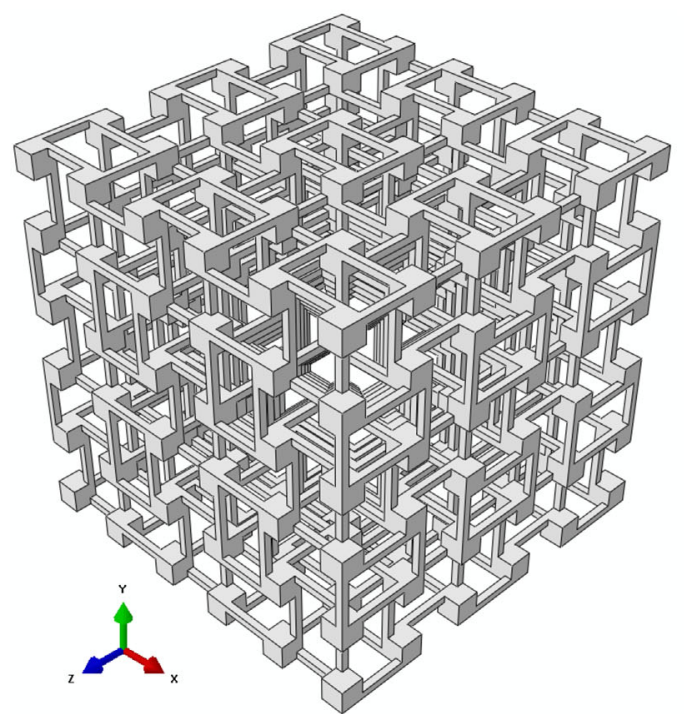

Figure 1. Geometrical parameters and topology of the 3D isotropic antitetrachiral unit cell with cubic nodes. 
are performed to verify the proposed theoretical models. Mechanical properties of the 3D anti-tetrachiral structure can be controlled and tuned with two independent dimensionless geometrical parameters, and the mechanical properties dependence on geometrical features are studied. The proposed 3D anti- tetrachiral structures can be employed for vibration attenuation, energy absorption and flexible engineering structure application.

\section{Mechanical Properties of 3D Isotropic Anti- Tetrachiral Metastructure}

In this paper, making use of the half-wave deformation mode of anti-chiral ligaments, 3D isotropic anti-tetrachiral structure consisting of cubic nodes and straight ligaments with square cross-sections are proposed, and the geometrical topology of the 3D isotropic anti-tetrachiral unit cell is shown in Figure 1, where $L, d$, and $t$ represent the ligament length, cubic node width, and wall thickness of the ligaments. For convenience, the geometrical parameters of the 3D isotropic anti-tetrachiral unit cell can be reduced to dimensionless parameters: $\alpha=L / d$ and $\beta=t / d$, respectively.

In the pioneering work on the in-plane mechanical properties of 2D hexachiral honeycomb performed by Prall and Lakes, ${ }^{[4]}$ the following five assumptions are proposed: 1 ) nodes (or circles) are considered rigid; 2) internal forces oriented in a direction perpendicular to the externally applied stress vanish; 3) internal forces are dictated by the observed kinematic behavior; 4) axial and shear deformations of the ligaments are neglected; and 5) all deflections are small. Similar to the works performed by Alderson el al., ${ }^{[2]}$ Prall et al. ${ }^{[4]}$ and Chen et al. ${ }^{[6]}$ the mechanical properties of the $3 \mathrm{D}$ isotropic anti-tetrachiral structure in Figure 1 are studied based on rigid node rotation and ligament bending deformation, the deformation modes of the 3D chiral structure are ligament bending dominated, the shearing, tension force, and deformation of the ligaments are not included for analysis.
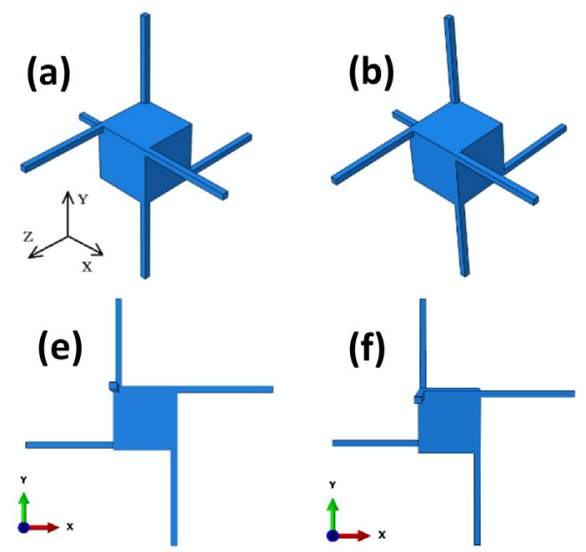

$\varepsilon_{x}=\varepsilon_{Y}=\varepsilon_{z}=\frac{2(d-t) \varphi}{(L+d)}$

As shown in Figure 2, when the 3D isotropic anti-tetrachiral structure is under uniaxial loading along $z$ direction, the cubic nodes will rotate by a small angle $\varphi$ off the $x, \gamma$, and $z$ direction equally, thus following deformation relations will be generated: $\varphi_{\mathrm{x}}=\varphi, \varphi_{\mathrm{y}}=\varphi$, and $\varphi_{\mathrm{z}}=\varphi$. The final strains $\varepsilon_{\mathrm{x}}, \varepsilon_{\mathrm{y}}$, and $\varepsilon_{\mathrm{z}}$ along the $x, y$, and $z$ directions can be expressed as

accordingly, the in-plane Poisson's ratio $v_{x y}$ can be calculated from its definition:

$v_{x z}=v_{y z}=-\frac{\varepsilon_{x}}{\varepsilon_{z}}=-\frac{\varepsilon_{y}}{\varepsilon_{z}}=-1$.

Then, the energy approach is employed to obtain the linear elastic modulus of the 3D chiral structures. It is assumed that the strain energy generated by a small strain $\varepsilon_{\mathrm{i}}$ along the $i$-th direction is identical to the elastic energy $W_{\text {lig }}$ stored in all the bending ligaments of the representative unit cell:

$\frac{1}{2} E_{i} \varepsilon_{i}^{2}=\frac{1}{V} \sum W_{\text {lig }}$

where $E_{i}$ and $V\left(V=8(L+d)^{3}\right)$ are the elastic modulus in $i$ direction and volume of the representative unit cell respectively. According to Euler-Bernoulli linear elastic beam theory, two equal and opposing moments $M$ acting on each end of the ligament produce an angular deflection $\varphi$, thus forming a half-wave deformation mode, and the strain energy within the ligaments can be therefore expressed as:

$W_{\text {lig }}=2 \int_{0}^{\varphi} M d \varphi=\frac{1}{2} \int_{0}^{L_{\mathrm{eff}}} \frac{M_{\mathrm{z}}^{2}}{E_{\mathrm{c}} I_{\mathrm{z}}} d l=\frac{E_{\mathrm{c}} t^{4} \varphi^{2}}{6 L_{\mathrm{eff}}}$,

where $L_{\text {eff }}=L$ is the effective length of the bending ligaments, $\varphi$ is the bending angle at the ligament end due to cubic rigid node rotation, and $E_{\mathrm{c}}$ is the elastic modulus of constituent material of the ligaments and cubic nodes.
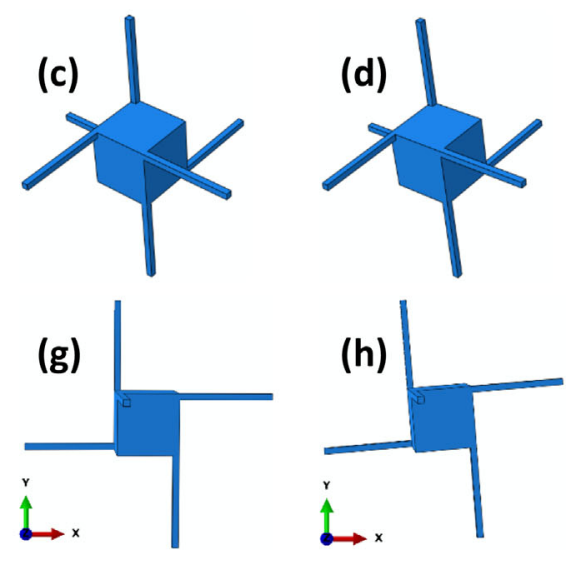

Figure 2. Top row shows the 3D representation of cubic node: a) original configuration without external loading condition; b) rotation about the $x$ axis for a small angle $\varphi ; c)$ additional rotation about the y axis for a small angle $\varphi$; d) additional rotation about the $z$ axis for a small angle $\varphi$, thus generate the final cubic node configuration. Bottom row shows the 2D projection of the cubic node onto the $x-y$ plane: $e$ ) original configuration without external loading condition; $f$ ) rotation about the $x$ axis for a small angle $\varphi$; g) additional rotation about the $y$ axis for a small angle $\varphi ; h$ ) rotation about the $z$ axis for a small angle $\varphi$. 
(a)

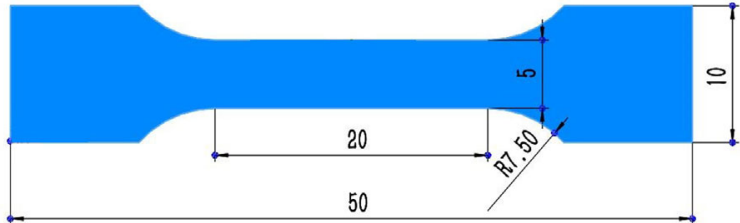

(b)

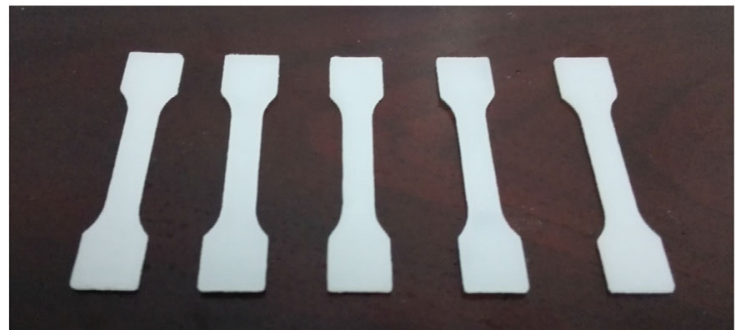

Figure 3. As fabricated tensile samples using nylon powder SLS technique: (a) Geometrical layout; (b) as-fabricated samples.

When the unit cell is under uniaxial tensile (or compression) loading along $z$ direction, energy expressions can be obtained by substituting Eqs. (1) and (4) into Eq. (3), and following equation can be generated:

$\frac{1}{2} E_{\mathrm{z}} \varepsilon_{\mathrm{z}}^{2}=8\left[\frac{E_{\mathrm{c}} t^{4}(L+d)^{2} \varepsilon_{\mathrm{z}}^{2}}{24 L(d+L)^{2}}+\frac{E_{\mathrm{c}} t^{4}(L+d)^{2} \varepsilon_{\mathrm{z}}^{2}}{24 L(d-t)^{2}}+\frac{E_{\mathrm{c}} t^{4}(L+d)^{2} \varepsilon_{\mathrm{z}}^{2}}{24 L(d-t)^{2}}\right] \frac{1}{8(L+d)^{3}}$

where $E_{\mathrm{z}}$ is the elastic modulus along $z$ direction of the 3D isotropic anti-tetrachiralstructure. Finally, Eq. (5) can be further reduced to the following dimensionless equation:

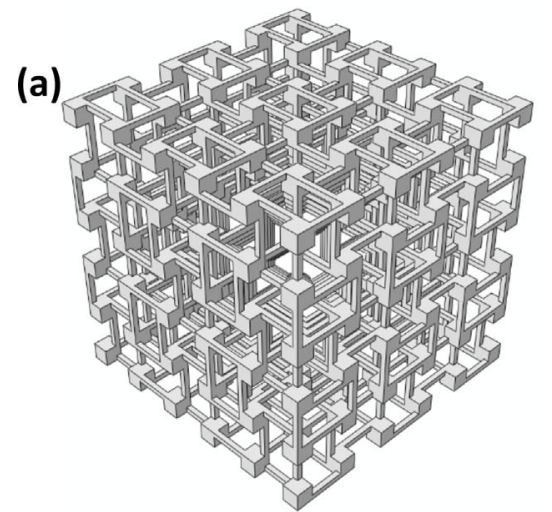

(c)

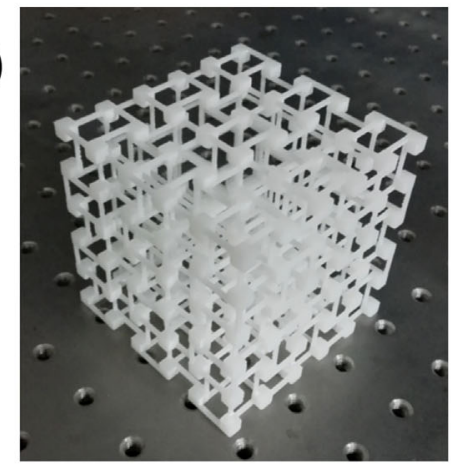

$E_{z}=\frac{E_{c} t^{4}}{4 L(L+d)(d-t)^{2}}$.

Due to the 3D isotropic topological design of anti-tetrachiral structure along $x, y$, and $z$ directions, mechanical properties of the $3 \mathrm{D}$ anti-tetrachiral structure are isotropic, and the modulus along $x, y$, and $z$ direction can be expressed as:

$E_{\mathrm{x}}=E_{\mathrm{y}}=E_{\mathrm{z}}=\frac{E_{\mathrm{c}} \beta^{4}}{4 \alpha(\alpha+1)(1-\beta)^{2}}$.

\section{Results}

\subsection{Comparison Between Theory, FEA, and Experimental Study}

In order to confirm the theoretical modulus of the3D isotropic anti-tetrachiral structures, uniaxial tensile samples are fabricated with additive manufacturing 3D printer $\mathrm{FS} 401^{\circledR}$, which is constructed by HuNan Farsoon High-Technology Co. Ltd. in HuNan Province of China, and the spatial resolution is $8 \mu \mathrm{m}$ along $x, y$, and $z$ directions. In total, five uniaxial tensile samples are fabricated with nylon powder Selected Laser Sintering (SLS) technique, and the as-fabricated 3D printed tensile samples are shown in Figure 3. The uniaxial tensile experiments are performed on an Instron ${ }^{\mathbb{R}} 5985$ machine at a displacement rate of $1 \mathrm{~mm} \mathrm{~min}-1$, and the force resolution of the loading cell is

(b)

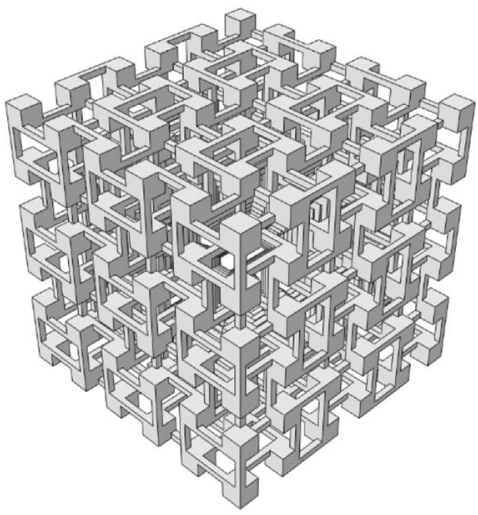

(d)

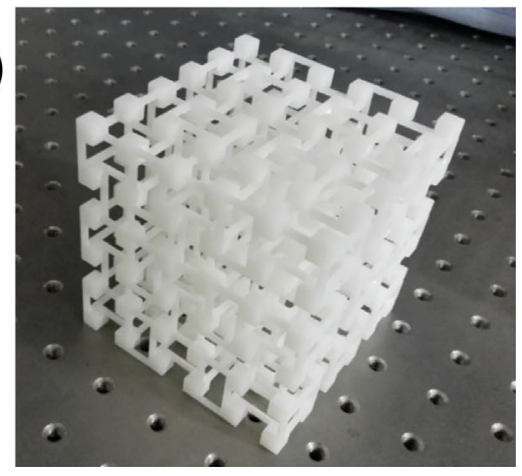

Figure 4. Geometrical parameters of 3D samples fabricated with SLS technique: (a) designed and (c) as-fabricated with $t_{1}=1.5 \mathrm{~mm}, d_{1}=5.0 \mathrm{~mm}$, $L_{1}=10 \mathrm{~mm}$; (b) designed and (d) as-fabricated with $t_{2}=2.0 \mathrm{~mm}, d_{2}=7.0 \mathrm{~mm}, L_{2}=10 \mathrm{~mm}$. 
Table 1. Modulus comparison between theory, FEA, and experimental studies.

\begin{tabular}{lccc}
\hline Samples & Theoretical results (MPa) & FEA results (MPa) & Average experimental results (MPa) \\
\hline Type $1\left(t_{1}=1.5 \mathrm{~mm}, d_{1}=5.0 \mathrm{~mm}, L_{1}=10 \mathrm{~mm}\right)$ & 0.11835 & 0.1642 & $0.1438 \pm 0.0134$ \\
Type $2\left(t_{2}=2.0 \mathrm{~mm}, d_{2}=7.0 \mathrm{~mm}, L_{2}=10 \mathrm{~mm}\right)$ & 0.1617 & 0.2032 & $0.1841 \pm 0.0232$ \\
\hline
\end{tabular}

$0.010 \mathrm{~N}$. Finally, the average elastic modulus of the 5 asfabricated tensile samples is: $E_{\mathrm{s}}=171.8 \mathrm{MPa}$, where the deviation of the modulus is: $\pm 1.24 \mathrm{MPa}$.

After harvesting the modulus of the constituent materials, two types of 3D anti-tetrachiral specimens with cubic nodes are fabricated through 3D nylon powder SLS additive manufacturing technique. As shown in Figure 4, the geometries of the two types of samples are: (a) Type 1 with $t_{1}=1.5 \mathrm{~mm}, d_{1}=5.0 \mathrm{~mm}$, and (b) Type 2 with $L_{1}=10 \mathrm{~mm} ; t_{2}=2.0 \mathrm{~mm}, d_{2}=7.0 \mathrm{~mm}$, and $L_{1}=10 \mathrm{~mm}$, respectively. Then, uniaxial compression experiments are performed on an Instron ${ }^{\mathbb{R}} 5985$ tensile machine at a displacement rate of $1 \mathrm{~mm} \mathrm{~min}^{-1}$ for these two types of samples, and the force resolution of the loading cell is $0.010 \mathrm{~N}$. In total, four specimens of the same geometrical parameters are fabricated and tested for each type of specimen, and the average experimental results are shown in Table 1.

Finite element analysis (FEA) was performed with Abaqus ${ }^{\mathbb{R}}$ standard for simulating the mechanical behaviors of these two types of 3D isotropic anti-tetrachiral samples under uniaxial compression loading conditions, where the finite element models and material properties are identical as corresponding experimental samples. The two types of FEA models are meshed with C3D10 tetrahedron element, the meshing size is $1.5 \mathrm{~mm}$ for Type 1 sample, and totally 102957 elements are generated; the meshing size is $2.0 \mathrm{~mm}$ for Type 2 sample, and totally 123059 elements are generated. Normal compression pressure along $Y$ direction is applied onto the upper cubic node surfaces, which were constrained from rotation. The bottom cubic node surfaces were constrained from rotation and translation along $y$ direction. To avoid rigid body movements of the whole model, nodes on the bottom node surfaces were constrained from translation displacement normal to the $y$ direction. All simulations were performed up to $1 \%$ nominal strain.

Nominal stress and strain of the 3D anti-tetrachiral structure in the $y$ direction was calculated from:

$\varepsilon_{\mathrm{y}}=\delta / L_{\mathrm{eff}} ; \sigma_{\mathrm{y}}=F / A_{\mathrm{eff}}$, where $\delta$ is the applied displacement onto the upper node surfaces, $F$ is the sum of the nodal reaction forces on the node surfaces to which displacement was applied, and $L_{\text {eff }}$ and $A_{\text {eff }}$ are the initial length along $y$ direction and cross-sectional area of the 3D anti-tetrachiral structure perpendicular to the $y$ direction, respectively. Finally, the modulus of the 3D antitetrachiral structure was calculated by:

$E=\sigma_{\mathrm{y}} / \varepsilon_{\mathrm{y}}$

Besides experimental and FEA studies of the modulus of the 3D isotropic anti-tetrachiral structure, theoretical modulus are produced with Eq. (7) for these two types of 3D anti-tetrachiral samples, and theoretical, experimental and finite element simulation modulus results are shown in Table 1 . It can be seen that the differences between theoretical, experimental and FEA results are acceptable, and theoretical results are lower than experimental and simulation results. This is because the ligaments shearing and tension deformation induced strain energy is not included in the theoretical model, and the cubic nodes are treated as rigid rotation nodes. Moreover, free surface effects in not included in the theoretical models due to missing ligaments. The experimental deformation results of the two types of $3 \mathrm{D}$ isotropic anti-tetrachiral structures at $\varepsilon_{\mathrm{z}}=1 \%$ axial compression loading strain are shown in Figure 5 , and the finite element simulated deformation fields of the two types of 3D anti-tetrachiral samples under $\sigma_{\mathrm{y}}=0.01 \mathrm{MPa}$ compression stress along the axial directions are shown in Figures 6 and 7. Through comparison between Figures 5, 6 and 7, it can be confirmed that the anti-tetrachiral ligaments of the $3 \mathrm{D}$ isotropic anti-tetrachiral samples will produce half-wave deformation mode in all directions.

Meanwhile, Poisson's ratios of the two types of 3D antitetrachiral samples during compression tests are analyzed. As shown in Figure 8, in order to reduce the free surface effects on the Poisson's ratio analysis for the 3D isotropic anti-tetrachiral structure, unit cell structure in the center is selected. As shown
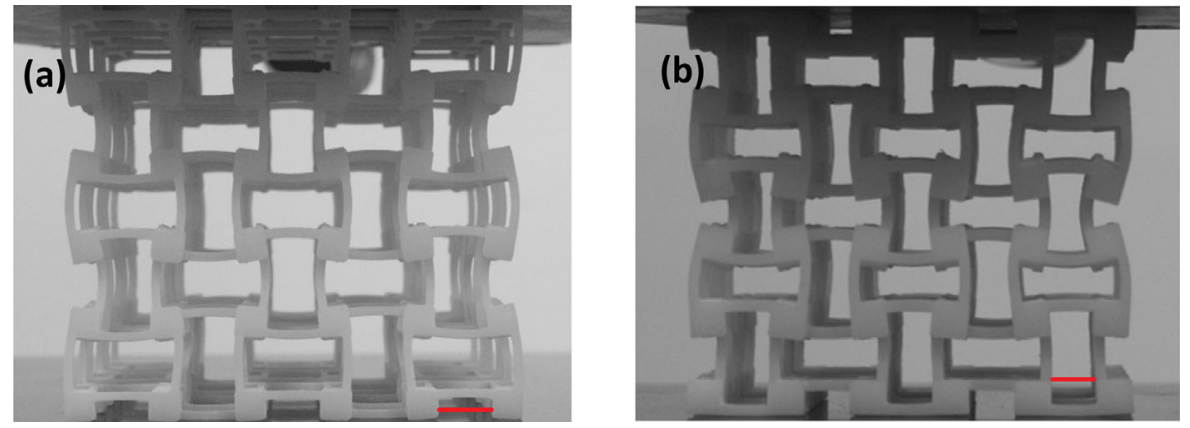

Figure 5. Experimental deformation after $\varepsilon_{\mathrm{z}}=1 \%$ axial loading: (a) $t_{1}=1.5 \mathrm{~mm}, d_{1}=5.0 \mathrm{~mm}, L_{1}=10 \mathrm{~mm}$; (b) $t_{2}=2.0 \mathrm{~mm}, d_{2}=7.0 \mathrm{~mm}, L_{2}=10 \mathrm{~mm}$. 
(a)

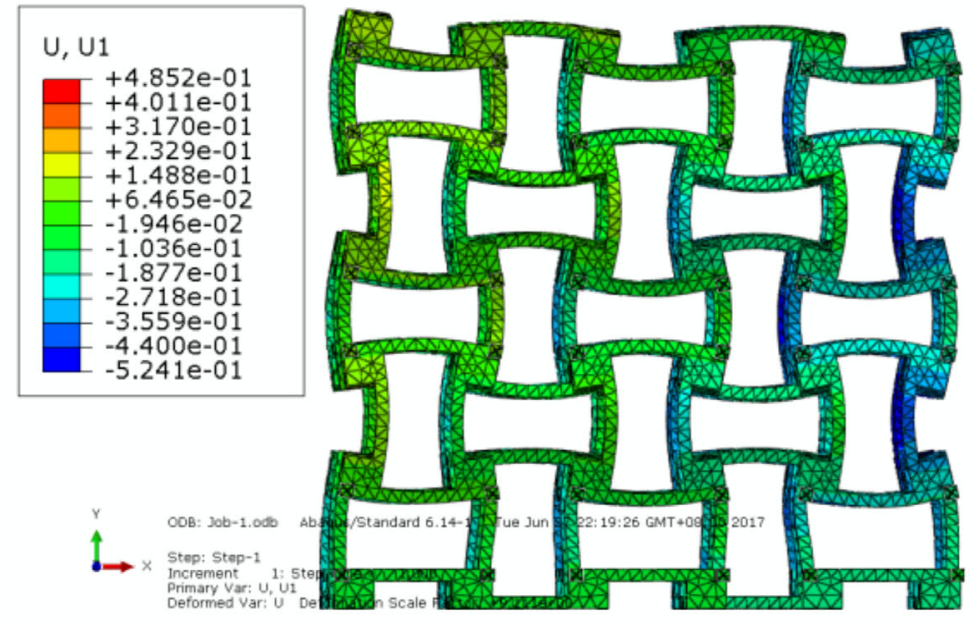

(b)

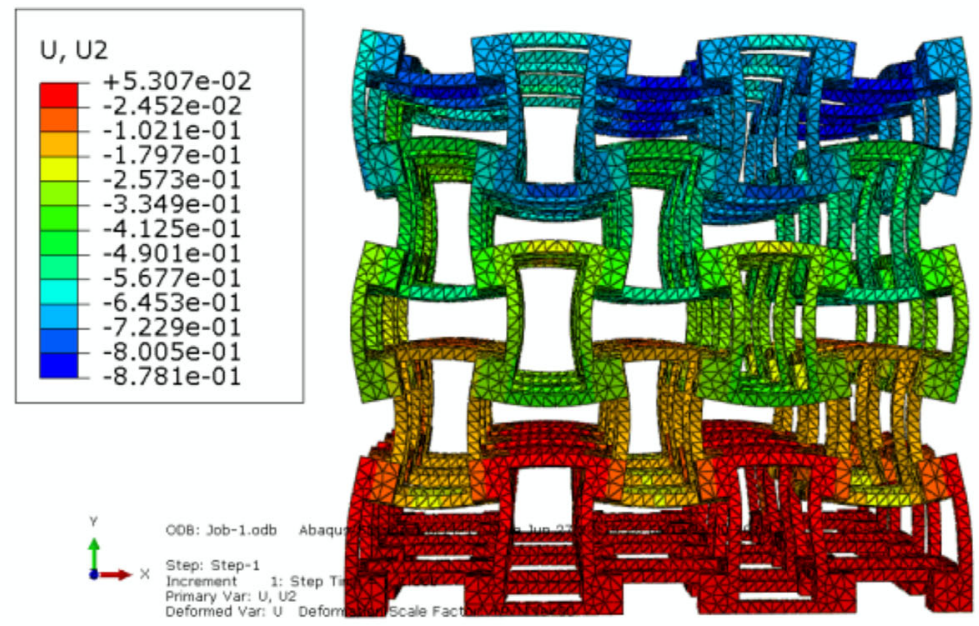

(c)

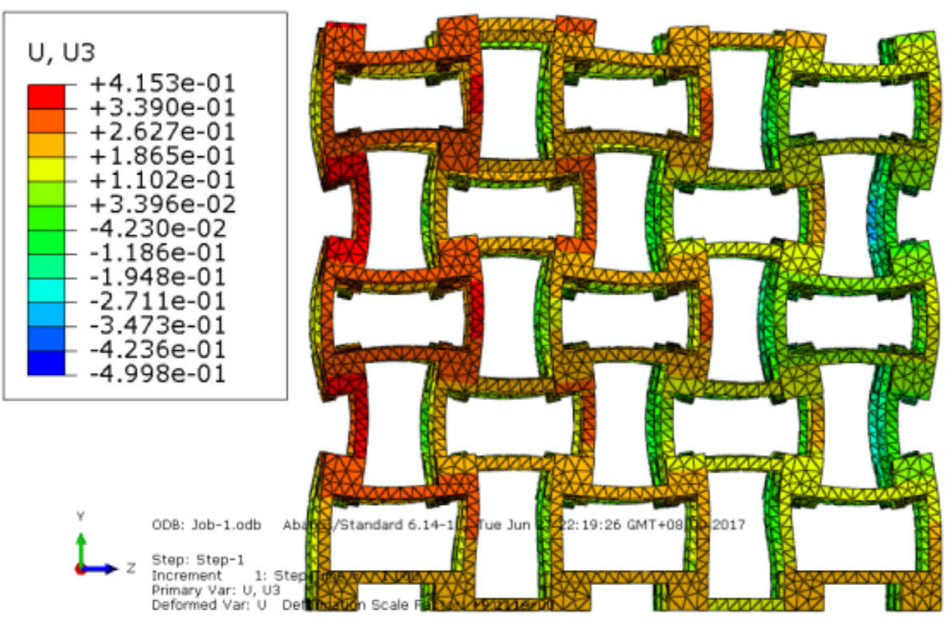

Figure 6. Elastic fields of Type $1\left(t_{1}=1.5 \mathrm{~mm}, d_{1}=5.0 \mathrm{~mm}, L_{1}=10 \mathrm{~mm}\right)$ under uniaxial normal stress $\sigma_{\mathrm{yy}}=0.01 \mathrm{MPa}$ along $y$ direction: a) displacement $\mathrm{U}$ along $x$ direction; b) displacement $\mathrm{V}$ along $\mathrm{y}$ direction; c) displacement $\mathrm{W}$ along $z$ direction. 


\section{ADVANCED

(a)

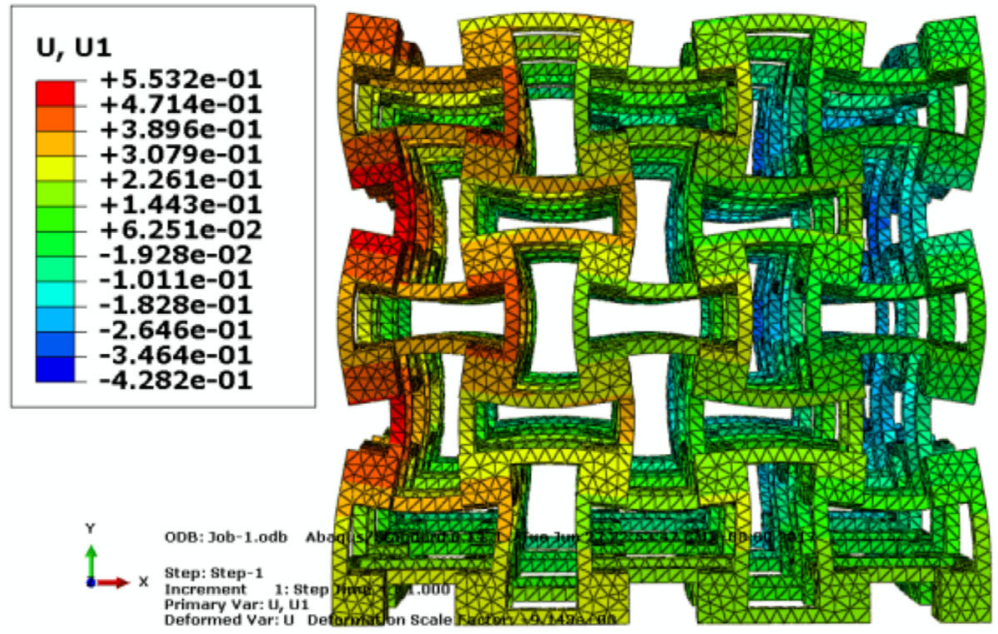

(b)

\begin{tabular}{|l|}
\hline U, U2 \\
\hline$+4.571 \mathrm{e}-02$ \\
$-4.195 \mathrm{e}-02$ \\
$-1.296 \mathrm{e}-01$ \\
$-2.173 \mathrm{e}-01$ \\
$-3.049 \mathrm{e}-01$ \\
$-3.926 \mathrm{e}-01$ \\
$-4.802 \mathrm{e}-01$ \\
$-5.679 \mathrm{e}-01$ \\
$-6.556 \mathrm{e}-01$ \\
$-7.432 \mathrm{e}-01$ \\
$-8.309 \mathrm{e}-01$ \\
$-9.185 \mathrm{e}-01$ \\
$-1.006 \mathrm{e}+00$ \\
\hline
\end{tabular}

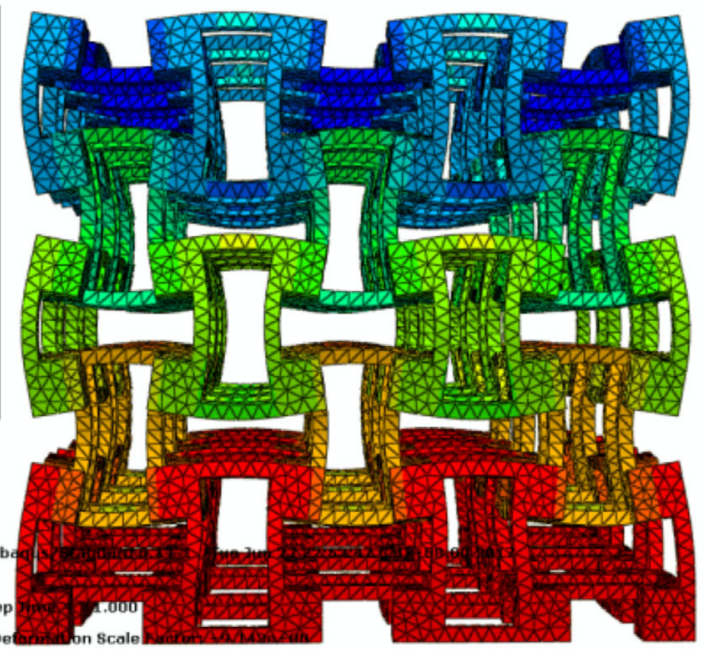

(c)

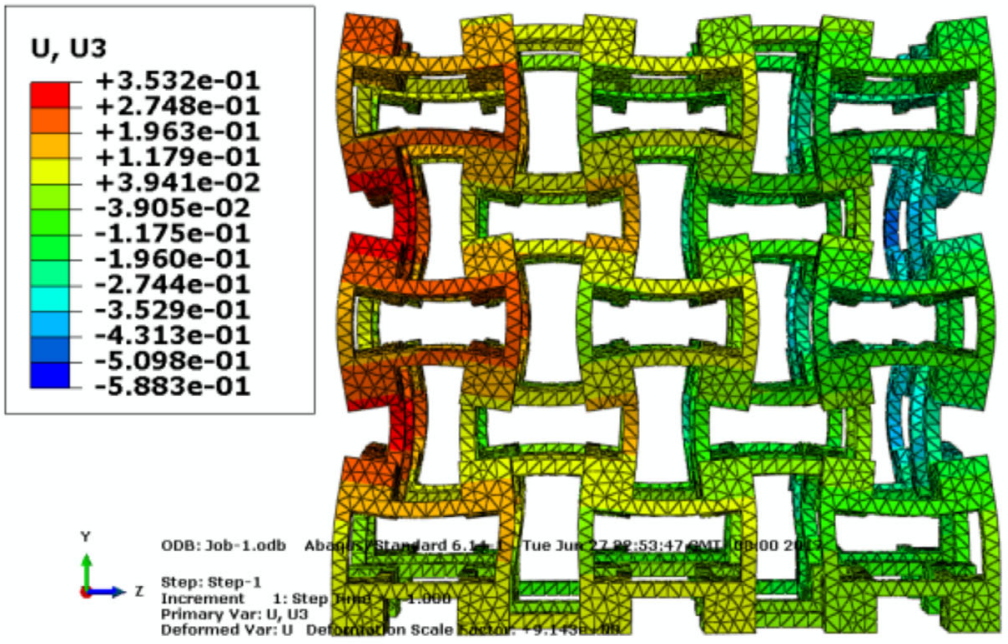

Figure 7. Elastic fields of Type $2\left(t_{2}=2.0 \mathrm{~mm}, d_{2}=7.0 \mathrm{~mm}, L_{2}=10 \mathrm{~mm}\right)$ under uniaxial normal stress $\sigma_{y y}=0.01 \mathrm{MPa}$ along $\gamma$ direction: a) displacement $\mathrm{U}$ along $x$ direction; b) displacement $\mathrm{V}$ along y direction; $\mathrm{c}$ ) displacement $\mathrm{W}$ along $z$ direction. 


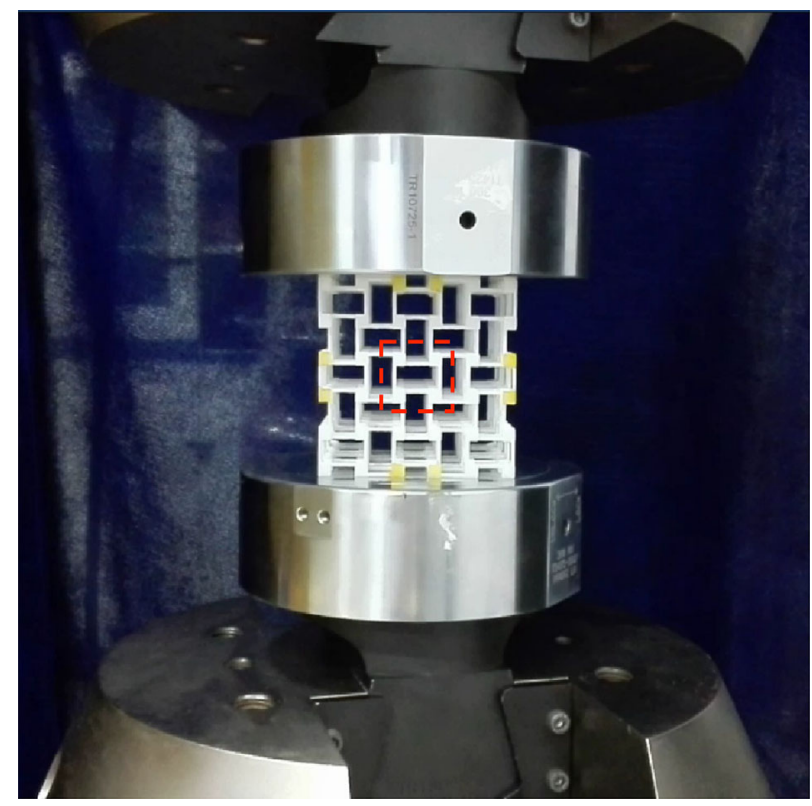

Figure 8. In-situ compression test of the 3D isotropic anti-tetrachiral structure, where red dotted line local region is selected for Poisson's ratio analysis.

in Figure 9a, four points A, B, C, and D on the dotted blue line along $x$ direction over the edges of cubic nodes are selected as marks for calculating the strain along $x$ direction; another four points $\mathrm{E}, \mathrm{F}, \mathrm{G}$, and $\mathrm{H}$ on the dotted blue line along $Y$ direction over the edges of cubic nodes are selected as marks for calculating the strain along $x$ direction. The middle points of $A B$, $\mathrm{CD}, \mathrm{EF}$, and $\mathrm{GH}$ can be calculated out through analyzing the gray value profiles in Figure 9b-c, the distances changes between the centers of $\mathrm{AB}$ and $\mathrm{CD}$ along $x$ direction, the centers of $\mathrm{EF}$ and $\mathrm{GH}$ along $\gamma$ direction during the deformation process can be calculated out, and the corresponding strains along $x$ and $y$ directions can be generated for Poisson's ratio calculation. Finally, the average experimental Poisson's ratio results during the compression test are shown in Table 2, it can be seen that the experimental Poisson's ratio results are close to the theoretical prediction $v=-1.0$ for both types of $3 \mathrm{D}$ antitetrachiral samples.

\subsection{Effects of Geometrical Parameters on the Mechanical Properties}

In this part, effects of dimensionless geometrical parameters on the mechanical properties of the $3 \mathrm{D}$ isotropic antitetrachiral structure are studied. As shown in Eq. (7), there are two dimensionless parameters $\alpha$ and $\beta$ employed for describing the mechanical properties of the 3D anti- tetrachiral structure. Assuming $\beta=0.15,0.20,0.25$, effects of $\alpha$ on the relative modulus $E / E_{\mathrm{s}}$ of the $3 \mathrm{D}$ isotropic anti-tetrachiral structure are shown in Figure 10, and it can be concluded that: with the increase of $\alpha$ at constant given $\beta$ value, the relative modulus $E / E_{\mathrm{s}}$ drops rapidly. It can be seen from Figure 10 that the mechanical properties of the proposed innovative 3D isotropic anti-tetrachiral structure can be adjusted in a quite
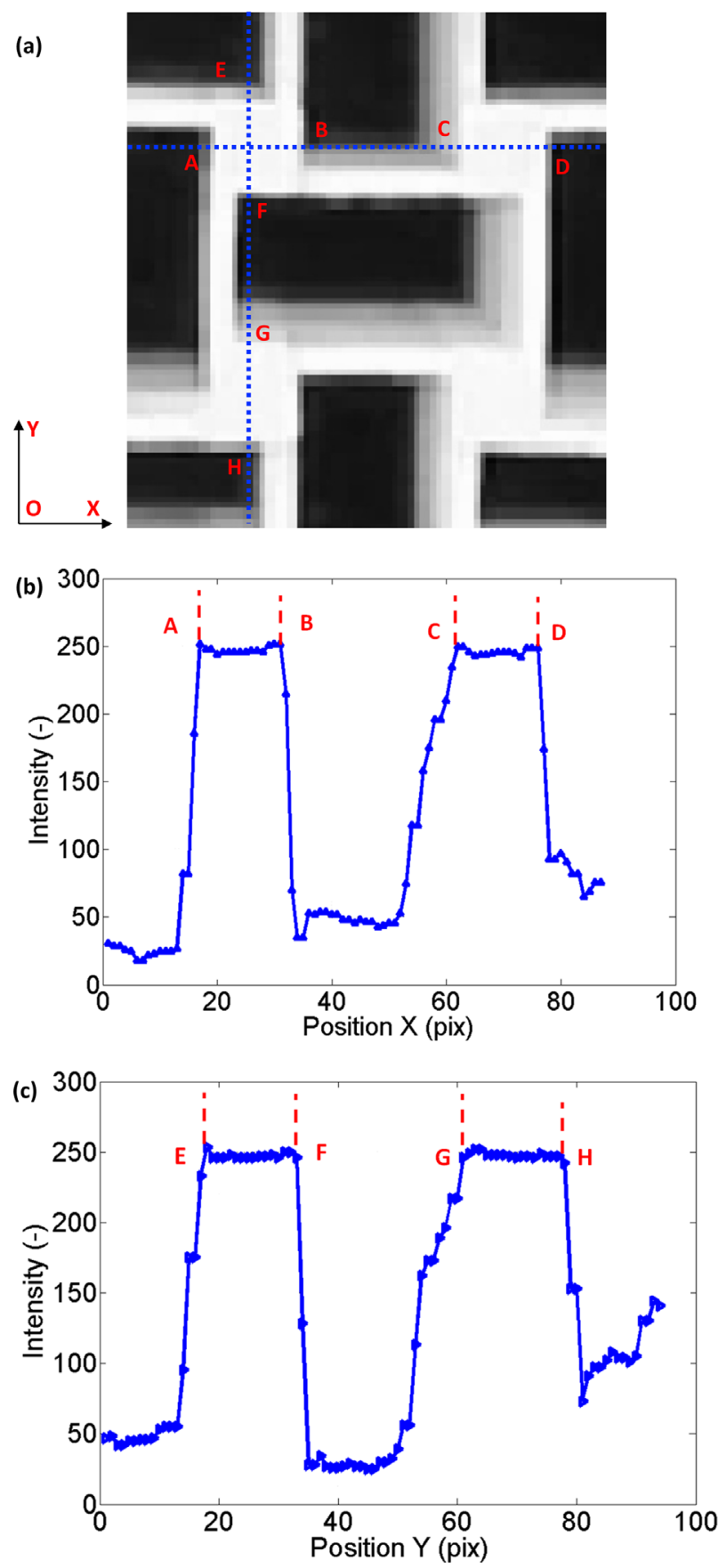

Figure 9. Calculation method of the Poisson's ratio during in-situ compression: (a) horizontal and vertical line across the node face center; (b) the gray value profile for calculating the strain along $x$ direction; and (c) the gray value profile for calculating the strain along $y$ direction.

Table 2. Experimental Poisson's ratio.

\begin{tabular}{lc}
\hline Samples & Average Poisson's ratio \\
\hline Type $1\left(t_{1}=1.5 \mathrm{~mm}, d_{1}=5.0 \mathrm{~mm}, L_{1}=10 \mathrm{~mm}\right)$ & $0.90 \pm 0.12$ \\
Type 2 $\left(t_{2}=2.0 \mathrm{~mm}, d_{2}=7.0 \mathrm{~mm}, L_{2}=10 \mathrm{~mm}\right)$ & $0.84 \pm 0.06$ \\
\hline
\end{tabular}




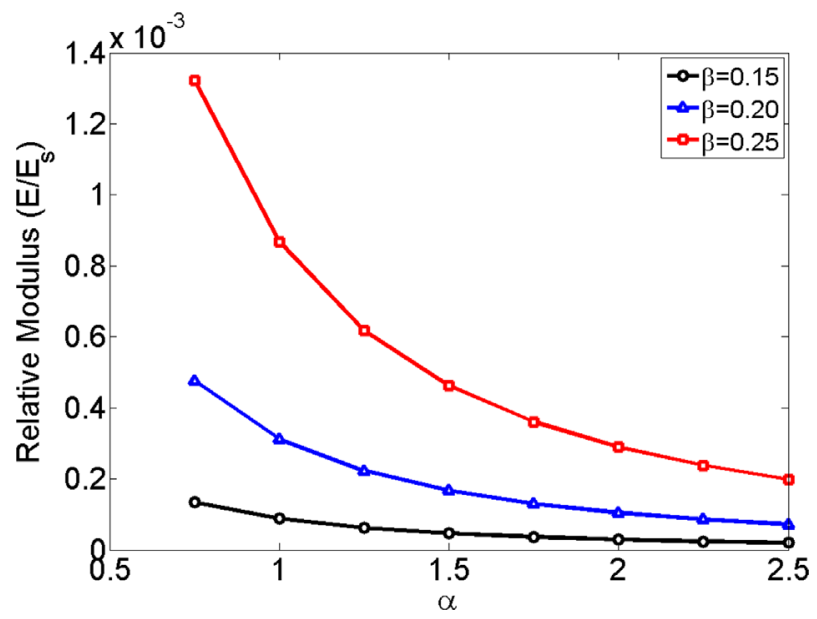

Figure 10. Relation between relative modulus $\left(E / E_{\mathrm{s}}\right)$ and dimensionless geometrical parameters $(\alpha, \beta)$.

large range, and can be used in flexible industrial components, biomedical devices, impact and blast-proof energy absorption components and reconfigurable soft robotics etc.

\section{Conclusion}

Based on rigid cubic node rotation and kinematic deformation geometrical relation, the analytical expressions for the modulus of 3D isotropic anti-tetrachiral structure with negative Poisson ratio are derived from strain energy analysis, comparisons between experiments, finite element analysis (FEA), and theoretical studies are performed for verification, and the mechanical properties of the $3 \mathrm{D}$ anti-tetrachiral structures can be controlled and tuned with two independent dimensionless geometrical parameters.

\section{Acknowledgements}

This research is supported by the National Natural Science Foundation of China (Grant no. 11702023), and the 2017 Opening fund of State Key Laboratory of Nonlinear Mechanics, Institute of Mechanics, Chinese Academy of Sciences.

\section{Conflict of Interest}

The authors declare no conflict of interest.

\section{Keywords}

3D anti-tetrachiral, auxetic, isotropic, modulus

Received: July 7, 2017

Revised: November 18, 2017

Published online: December 18, 2017

[1] L. Kelvin, Baltimore Lectures on Molecular Dynamics and the Wave Theory of Light. C.J. Clay and Sons, London 1904.

[2] N. Novak, M. Vesenjak, Z. Ren, Stroj. Vestn. J. Mech. E 2016, 62, 485.

[3] A. Alderson, K. L. Alderson, D. Attard, K. E. Evans, R. Gatt, J. N. Grima, W. Miller, N. Ravirala, C. W. Smith, K. Zied, Composites Sci. Technol. 2010, 70, 1042.

[4] D. Prall, R. S. Lakes, Int. J. Mech. Sci. 1997, 39, 305.

[5] D. Mousanezhad, B. Haghpanah, R. Ghosh, A. M. Hamouda, H. N. Hashemi, A. Vaziri, Theor. Appl. Mech. Lett. 2016, 6, 81.

[6] Y. J. Chen, F. Scarpa, Y. J. Liu, J. S. Leng, Int. J. Solids Struct. 2013, 50, 996.

[7] A. Spadoni, M. Ruzzene, J. Mech. Phys. Solids 2012, 60, 156.

[8] A. Spadoni, Application of chiral cellular materials for the design of innovative components. Georgia Institute of Technology, 2008.

[9] A. Bacigalupo, M. L. Bellis, Composites Struct. 2015, 131, 530.

[10] A. Bacigalupo, L. Gambarotta, Composites Struct. 2014, 116, 461.

[11] X. N. Liu, G. L. Huang, G. K. Hu, J. Mech. Phys. Solids 2012, 60, 1907.

[12] Y. Chen, X. N. Liu, G. K. Hu, Q. P. Sun, Q. S. Zheng, Proc. R. Soc. A 2013, 470A, 20130734.

[13] H. M. Li, Y. B. Ma, W. B. Wen, W. W. Wu, H. S. Lei, D. N. Fang, J. Appl. Mech.,Trans. ASME 2017, 84, 081006.

[14] W. W. Wu, Y. Tao, Y. Xia, J. K. Chen, H. S. Lei, L. J. Sun, D. N. Fang, Extreme Mech. Lett. 2017, 16, 18.

[15] X. N. Liu, G. K. Hu, Stroj. Vestn. J. Mech. E. 2016, 62, 403.

[16] A. Spadoni, M. Ruzzene, ASME J. Vib. Acoust. 2006, 182, 616.

[17] A. Spadoni, M. Ruzzene, F. Scarpa, Smart Struct. Mater. Conference, San Diego, CA March 2005, Proc. SPIE 2005, 5764. https//doi.org/ $10.1117 / 12.600021$.

[18] O. Abdeljaber, O. Avci, D. J. Inman, J. Sound Vib. 2016, 369, 50.

[19] F. Scarpa, M. R. Hassan, M. Ruzzene, Proc. SPIE 2006, 6170, 61700W.

[20] M. R. Hassan, F. Scarpa, M. Ruzzene, N. A. Mohammed, Mater. Sci. Eng. A 2008, 481-482, 654.

[21] A. Spadoni, M. Ruzzene, J. Mech. Mater. Struct. 2007, 2, 965.

[22] P. R. Budarapu, S. Sastry, Y. B. R. Natarajan, Front. Struct. Civ. Eng. 2016, 10, 394.

[23] Y. Ma, F. Scarpa, D. Zhang, B. Zhu, L. Chen, J. Hong, Smart Mater. Struct. 2013, 22, 084012.

[24] C. S. Ha, M. E. Plesha, R. S. Lakes, Smart Mater. Struct. 2016, 25, 054005.

[25] C. S. Ha, M. E. Plesha, R. S. Lakes, Phys. Status Solidi B 2016, 253B, 1243. 